\title{
Changes to glaucoma surgery patterns during the COVID-19 pandemic: A shift toward less invasive procedures
}

\author{
Natalia Dub ${ }^{1}$, Kinga Gołaszewska ${ }^{1}$, Emil Saeed ${ }^{1}$, Diana Anna Dmuchowska ${ }^{1}$, Iwona Obuchowska ${ }^{1}$ and Joanna \\ Konopińska ${ }^{1, *}$
}

\author{
1Department of Ophthalmology, Medical University of Bialystok, 15-276 Bialystok, Poland \\ * Correspondence: joannakonopinska@o2.pl; Tel.: +48-857468372
}

Abstract:

The aim of the study was to compare the quantity, type of anti-glaucoma surgeries, and the disease stage before and during the coronavirus disease pandemic. This was a retrospective, single-center consecutive case series audit that included medical records of patients who underwent glaucoma surgery at the University Hospital in Białystok between September 4, 2018, and March 3, 2020 (prepandemic group) and compared with those of glaucoma patients treated between March 4, 2020, and September 4, 2021 (pandemic group). Adult patients with primary or secondary open-angle or closed-angle glaucoma who underwent anti-glaucoma surgery were included in this study. Finally, 534 operated eyes (362 and 172 eyes operated on before and during the pandemic, respectively) were examined. The number of anti-glaucoma surgeries was halved during the pandemic compared to a similar pre-pandemic period, with a significant difference in the kind of procedure between the two groups $(p<0.001)$. The most common procedures in the pre-pandemic group were Ex-Press implantation $(33.7 \%)$ and trabeculectomy $(31.5 \%)$. Within the pandemic group, half of the eyes underwent trabeculectomy (50.0\%), followed by Preserflo microshunt (11.6\%), iStent $(8.7 \%)$ and transcleral cyclophotocoagulation (TSCP), (8.7\%). A significant difference in the average IOPs was revealed among patients who qualified for surgery $22.21 \pm 7.83 \mathrm{mmHg}$ in the pre-pandemic group and $25.16 \pm 9.48 \mathrm{mmHg}$ in the pandemic group; $p<0.001$ ). In conclusion the COVID-19 pandemic is associated with a decrease in the number of extended procedures and an increase in the number of short procedures performed, such as TSCP and MIGS.

Keywords: glaucoma surgery; SARS-CoV-2 pandemic; minimally invasive glaucoma surgery; glaucoma surgery patterns; COVID-19; trabeculectomy; canaloplasty; iStent

\section{Introduction}

Glaucoma is a leading cause of blindness worldwide, affecting 76 million people in 2020 [1]. This number is expected to increase by 40 million in the next few years. In Poland, with a population of almost 38 million, the number of patients affected by glaucoma has been estimated at 800,000 [16]. Glaucoma treatment remains a challenge, and treatment discontinuation may result in an increased number of patients with irreversible vision loss. 
The first unexplained case of acute pneumonia occurred in November 2019 in Wuhan, China [2] and was soon thereafter associated with severe acute respiratory syndrome coronavirus 2 (SARS-CoV-2); the resulting disease is now known as the coronavirus disease 2019 (COVID-19) [25]. Due to the rapid and global spread of the disease, the World Health Organization proclaimed the outbreak as a pandemic on March 11, 2020. In Poland, the first case of COVID-19 was reported on March 4, 2020, and the first national lockdown was declared on March 23, 2020 [3,4].

The pandemic has forced a reorganization of healthcare systems worldwide, including the mobilization and prioritization of resources to treat COVID-19 patients. To limit disease spread and to protect resources, many ophthalmic societies provided guidelines for classifying procedures as urgent and non-urgent [5], with the latter group's procedures being postponed [6,7]. Certain ophthalmology departments were converted into COVID19 wards, admitting only SARS-CoV-2-positive patients and limiting access to ophthalmic treatment. The fear of infection among older adults and patients with comorbidities resulted in delays when seeking treatment for cataracts or chronic glaucoma [8].

The Ophthalmology Department of Medical University in Białystok, Poland, is a reference center for most patients affected by glaucoma in the Podlasie voivodeship (population of approximately 1.2 million people). It is the only hospital in this region that offers surgical treatment for glaucoma. Despite the restrictions placed on elective surgeries during the COVID-19 pandemic, antiglaucoma surgeries were classified as urgent and were regularly performed. The Royal College of Ophthalmology provided recommendations for qualifying glaucoma patients for surgical treatment [9], including those needing immediate and non-immediate surgery, based on intraocular pressure (IOP), extent of vision and visual field losses, disease progression rate, and suitability of other treatments. However, these recommendations did not address surgery or anesthesia types, both of which remained at the surgeon's discretion.

Few studies have examined the impact of the COVID-19 pandemic on ophthalmology departments and ophthalmic outpatient clinical activities. Despite generalized recommendations on safety during the pandemic [10-12], there has been a lack of detailed clinical studies on the impact of the SARS-CoV-2 pandemic on outcomes in patients with acute and chronic glaucoma. Rajendrababu et al. [13] made a comparison between emergency glaucoma patients treated in a tertiary eye care center in Madurai, South India, both before and during the COVID-19 lockdown. Meanwhile, Holland et al. [10] examined the impact of pandemic-related changes in glaucoma surgical practices in the United Kingdom. A separate study from the Tongji Hospital in Wuhan City, China, reported that glaucoma surgery was the most frequent subspecialty surgery performed during the COVID19 pandemic [14].

Given the lack of evidence on the impact of the COVID-19 pandemic on glaucoma treatment, this study aimed to compare surgical glaucoma care before and during the pandemic, including the quantity and type of anti-glaucoma procedures performed and the stage of the disease. To the best of our knowledge, our paper is the first report from a Central Eastern European referral center and might help to plan glaucoma surgery strategies in other cities and countries. These findings may help establish guidelines for the management of glaucoma patients during future outbreaks.

\section{Materials and Methods}

This was a retrospective, single-center consecutive case series audit that included patients with the International Classification of Diseases and Tenth Revision final diagnosis codes of B11 and B98, which encompass glaucoma surgery. Data of patients who underwent glaucoma surgery at the University Hospital in Białystok between September 4, 2018, and March 3, 2020, were extracted from their medical records and compared with those of glaucoma patients treated between March 4, 2020, and September 4, 2021. All adult patients with primary or secondary open-angle or closed-angle glaucoma who underwent anti-glaucoma surgery in the periods of interest were included in this study. 
Finally, 534 operated eyes (362 and 172 eyes operated on before and during the pandemic, respectively) were examined. All patients underwent a full ophthalmic examination prior to surgery to confirm the diagnosis of glaucoma. The ophthalmic examination included the assessment of best corrected visual acuity (BCVA) using the Snellen charts; IOP measurement by Goldmann applanation tonometry; assessment of the anterior segment of the eye by slit lamp, including ocular lens opacification severity, as determined by the Lens Opacification Classification Scale III; gonioscopy by Goldmann three-mirror lens; assessment of optic nerve disc by Volk lens, considering glaucoma-specific changes, and optical coherence tomography (OCT) (Heidelberg Engineering Heidelberg, Germany) of the optic nerve head and retinal nerve fiber layer [15].

In the period before the SARS-CoV-2 pandemic, each patient routinely underwent the 24-2 visual field examination (Humphery Field Analyzer, Zeiss Medical Technology, USA.) During the COVID-19 pandemic, when social distancing measures were introduced, examinations were conducted according to the disease spread containing guidelines [16,17]. Consequently, visual field examination was performed only in cases in which optical OCT measurements of the optic nerve head and retinal nerve fiber layer were insufficient to assess the severity of glaucomatous optic neuropathy.

The primary outcomes included the number of antiglaucoma surgeries performed and pre-surgical IOP values. The secondary outcomes included BCVA values, the extent of glaucomatous damage on OCT findings, and the type of surgery performed.

The study protocol adhered to the principles of the Declaration of Helsinki and was approved by the Bioethics Committee of the Medical University of Białystok (APK.002.87.2021). The informed consent requirement was waived due to the retrospective nature of this study. This study abided by the relevant data protection laws.

\subsection{Surgical technique}

Surgical procedures were performed as one-day procedures under local retrobulbar anesthesia by two experienced surgeons (JK and ES). Patients diagnosed with clinically significant cataracts (according to the Lens Opacification Classification Scale III) underwent a combined procedure of antiglaucoma surgery with simultaneous phacoemulsification. Patients with open-angle glaucoma qualified for one of the following surgical interventions: trabeculectomy, Ex-Press implantation, canaloplasty, and deep sclerectomy; patients with closed-angle glaucoma qualified for trabeculectomy. Patients with early-stage glaucoma or intermediate glaucomatous neuropathy underwent stent implantation. Traditional penetrating glaucoma surgeries were performed in patients with severe glaucomatous optic neuropathy.

During the pandemic, all patients underwent polymerase chain reaction testing for SARS-CoV-2 infection 2 days before the surgery, which was covered by the National Health Fund. Patients were then placed in self-isolation until admission for surgery. Since April 1, 2021, all vaccinated patients and patients that recovered from COVID-19 in the previous 3 months were exempt from the testing requirement.

\subsection{Statistical analysis}

Statistical analysis was performed using the R package, version 4.1.0 (Foundation for Statistical Computing, Vienna, Austria). Nominal variables are presented as counts (\%), while continuous variables are presented as mean \pm standard deviation (SD) or median (range), depending on data distribution. The normality of the distribution assumption was assessed using the Shapiro-Wilk test, data skewness, and kurtosis indicators based on the visual assessment of histograms. Between-group comparisons were performed using the chi-square test and Fisher exact test for categorical variables; the t-test and Mann-Whitney $\mathrm{U}$ test were used for comparisons of continuous variables, depending on data distribution. In addition, the mean or median difference and its $95 \%$ confidence interval were calculated. A significance level of $\alpha=0.05$ was used, and all tests were two-sided.

\section{Results}


A total of 306 and 149 patients were included in the pre-pandemic and pandemic groups, respectively. Sex distribution was similar in both groups $(p=0.939)$. The median age values in the pre-pandemic and pandemic groups were 73.0 and 70.0 years, respectively $(p=0.212)$ (Table 1$)$. 
Table 1. Clinical characteristics of patients with glaucoma, operated on before and during the COVID-19 pandemic

\begin{tabular}{llll}
\hline & Pre-pandemic group & Pandemic group & $p$-value \\
\hline Number of patients (operated & $306(362)$ & $149(172)$ & $<0.001$ \\
eyes) & & &
\end{tabular}

$$
\text { Sex, n }(\%)
$$

\begin{tabular}{clll}
\hline \multicolumn{1}{c}{ Male } & $130(42.5)$ & $62(41.6)$ & 0.939 \\
\cline { 1 - 2 } Female & $176(57.5)$ & $87(58.4)$ & 0.212 \\
\hline Age, median (range) ${ }^{*}$, years & $73.00(32-95)$ & $70.00(32-90)$ & $<0.001$ \\
\hline Number of surgical procedures & 354 & 172 & \\
\hline
\end{tabular}

Procedures by type, number (\%)

\begin{tabular}{|c|c|c|c|}
\hline Trabeculectomy & $114(31.5)$ & $86(50.0)$ & $<0.001$ \\
\hline Canaloplasty & $66(18.2)$ & $9(5.2)$ & $<0.001$ \\
\hline iStent & $33(9.1)$ & $15(8.7)$ & \\
\hline Ex-Press & $122(33.7)$ & $11(6.4)$ & \\
\hline Deep sclerectomy & $19(5.2)$ & $15(8.7)$ & \\
\hline Ahmed Valve & $0(0.0)$ & $1(0.6)$ & \\
\hline Microshunt Preserflo & $0(0.0)$ & $20(11.6)$ & \\
\hline TSCP* & $0(0.0)$ & $15(8.7)$ & \\
\hline Reoperations & $8(2.2)$ & $0(0.0)$ & \\
\hline
\end{tabular}

Phacoemulsification (overall), number $(\%)$

\begin{tabular}{|c|c|c|c|}
\hline Yes & $180(50.8)$ & $69(40.1)$ & 0.026 \\
\hline No & $174(49.2)$ & $103(59.9)$ & \\
\hline
\end{tabular}

*TSCP- transscleral cyclophotocoagulation

Groups were compared using the chi-square test (sex, phacoemulsification/all procedures/, phacoemulsification/all eyes), Mann-Whitney U test (age), and Fisher exact test (procedures by type, Trabeculectomy vs other procedures). 
The total number of procedures in the pre-pandemic and pandemic groups was 354 (354 eyes in 306 patients) and 172 (172 eyes in 149 patients), respectively. The most common procedure types in the pre-pandemic group were Ex-Press implantation (33.7\%) and trabeculectomy (31.5\%). Canaloplasty was relatively less common (18.2\%). IStent and deep sclerectomy were used in $<10 \%$ of the pre-pandemic patients. Ahmed valve implantation, microshunt Preserflo, and transscleral cyclophotocoagulation (TSCP) were not performed in patients from the pre-pandemic group. In addition, eight patients in the prepandemic group underwent reoperation (including one case of Ex-Press explantation due to extrusion, four cases of trabeculectomy revisions, and three cases of laser goniopuncture after deep sclerectomy).

In the pandemic group, $50.0 \%$ of the eyes underwent trabeculectomy, while a Preserflo microshunt was used in $11.6 \%$ of the cases. IStent, sclerectomy, and TSCP were each used in $8.7 \%$ of the eyes. Ex-Press procedure was performed in $6.4 \%$ of eyes, while canaloplasty was performed in $5.2 \%$ of eyes. One eye underwent the Ahmed procedure. The distribution of procedure types differed between the groups $(p<0.001)$ (Table 1$)$.

Before the pandemic, combined anti-glaucoma surgery with phacoemulsification was performed in $50.8 \%$ of all cases; the corresponding value during the pandemic was $40.1 \%(p=0.026)$ (Table 1 .)

Groups were compared using the chi-square test (sex, phacoemulsification/all procedures/, phacoemulsification/all eyes), Mann-Whitney U test (age), and Fisher exact test (procedures by type, Trabeculectomy vs other procedures).

The BCVA values were non-normally distributed; thus, they were reported as the median value 0.6 (range $0-1$ ) in both groups $(p=0.263)$. The mean IOP was $22.21(\mathrm{SD}=7.83)$ $\mathrm{mmHg}$ and $25.16(\mathrm{SD}=9.48) \mathrm{mmHg}$ in the pre-pandemic and pandemic groups, respectively $(p<0.001)$. There was a between-group difference in the OCT values $(p=0.021)$. The average OCT levels in the pre-pandemic and pandemic groups were $\mathrm{M}=57.77$ ( $\mathrm{SD}=19.63)$ and $\mathrm{M}=62.51(\mathrm{SD}=17.41)$, respectively (Table 2).

8 
Table 2. Comparison of best corrected visual acuity, intraocular pressure, and optical coherence tomography findings between patients operated on before and

\begin{tabular}{lllll}
\hline & Pre-pandemic group & Pandemic group & MD (95\% CI) & $p$-value \\
\hline BCVA $^{1}$ (Snellen) & $0.60(0-1)$ & $0.60(0-1)$ & $0.00(0.00 ; 0.00)$ & 0.263 \\
\hline IOP (mmHg) & $22.21 \pm 7.83$ & $25.16 \pm 9.48$ & $-2.95(-4.59 ;-1.32)$ & $<0.001$ \\
\hline OCT (M) & $57.77 \pm 19.63$ & $62.51 \pm 17.41$ & $4.75(0.72 ; 8.77)$ & 0.021 \\
\hline
\end{tabular}

Data are presented as mean $\pm \mathrm{SD}$ or median (range) ${ }^{1} . \mathrm{MD}$, mean or median difference (pre-pandemic minus pandemic) with $95 \%$ confidence interval (CI). $p$-value

- t-test or Mann-Whitney U test. BCVA, best corrected visual acuity; IOP, intraocular pressure; OCT, optical coherence tomography 


\section{Discussion}

This study has shown the negative impact of the COVID-19 pandemic in reducing the number of surgical procedures. During the pandemic, the number of anti-glaucoma surgeries performed at our clinic was half of those performed before the outbreak. Patients that qualified for surgeries during the pandemic had significantly higher IOP values than those that qualified before the pandemic. However, OCT examinations revealed that increased IOP did not affect the pathological stage of glaucomatous neuropathy. This may be due to patients not self-administering the IOP-lowering eyedrops; however, the study period was too short to reflect any morphological changes in the optic nerve. We also observed changes in the type of anti-glaucoma surgical procedures conducted during the pandemic. The number of procedures with long-lasting effects, including canaloplasty, decreased and that of minimally invasive glaucoma surgeries (MIGS), including Preserflo Micro Shunt implantation and TSCP, increased, likely due to reduced operative time. Moreover, the frequency of combined phacoemulsification and antiglaucoma surgeries decreased, and that of glaucoma surgery alone increased, likely in an attempt to save time and to avoid a potentially aerosol-generating procedure [24]. This approach is consistent with the guidelines for glaucoma patient care during the COVID-19 pandemic, which recommends single procedures and those that are associated with a relatively short followup [17]. The most frequently performed procedure in both periods was trabeculectomy, which is associated with its high success rate [9]. A significant increase in the frequency of MIGS is consistent with the long-term trend in the increasing uptake of minimally invasive procedures, including early-stage glaucomatous neuropathy care. The present findings are consistent with those of Rajendrababu et al. [18], who reported a significant decrease in the number of incisional glaucoma surgeries performed in favor of TSCP (by 2.8 times) and phacoemulsification alone (by 4.3 times). An increase in the number of performed phacoemulsifications as anti-glaucoma procedures may be associated with the increase in lens-induced glaucoma, which has been referred to as a reduction of elective cataract surgeries. A survey of glaucoma surgeons in the United Kingdom showed a change in patient attitudes toward glaucoma surgery after the pandemic outbreak [10]. Before the pandemic, the most popular procedure, as in our study, was trabeculectomy ( $87 \%$ as the procedure of choice). In the above-mentioned study, up to $60 \%$ of the surgeons declared modifications to their glaucoma surgery practice during the COVID-19 pandemic, mainly by reducing the number of performed trabeculectomies and by preferring TSCP as the most common alternative method. The reasons for these changes included a shorter postoperative follow-up period, increased procedural efficiency and safety, improved long-term outcomes, reduced operative time, less anesthesia burden, and fewer interventions in the postoperative period [10]. Despite these advantages, the long-term hypotensive effects of this method remain unclear.

The reduced number of procedures may also be accounted for by fewer patients electing for treatment due to the fear of infection among older adults and patients with comorbidities. Subathra et al. [17] reported a reduction in patient visits at a tertiary eye care center in South India during the pandemic; barriers to access included lockdown restrictions, transport problems, and fear of infection. In contrast, Mylona et al. [13], who conducted their study on the Greek population, found lower adherence to pandemic restrictions among patients who were older and had lower levels of education. In the present study, the lower number of referred patients might have been caused by the increased number of telephone counseling instead of traditional examination and the termination of routine outpatient visits.

This study had limitations. First, it was a single-center study, which may have been affected by the surgeons' preferences for particular techniques, which may also vary among centers. Second, we did not analyze cases in which phacoemulsification was performed as an anti-glaucoma treatment (patients with closed-angle glaucoma). It remains unclear how a substantial reduction in the number of performed visual field examinations 
impacts glaucoma management, as it is the gold standard for the diagnosis and treatment of patients with glaucoma and cannot be replaced by an OCT examination [5]. Moreover our findings may not be sufficiently generalizable, given that they were obtained from a single center. More similar studies analyzing the structure of antiglaucoma procedures during pandemic from other centers are needed. Future studies should also examine whether this factor affects the long-term quality of vision in glaucoma patients.

\section{Conclusions}

Despite diverting healthcare resources to the treatment of patients with COVID-19 during the SARS-CoV-2 pandemic, glaucoma treatment remained relatively accessible. This treatment should be provided safely and responsibly and in compliance with the relevant guidelines, including any infection prevention measures and adaptations to surgical procedures. The COVID-19 pandemic is associated with a decrease in the number of extended procedures and an increase in the number of short procedures performed, such as TSCP and MIGS. This shift may have reduced the number of required postoperative visits and interventions. The analysis of trends in glaucoma surgery during the COVID19 pandemic may help the ongoing development of treatment guidelines that inform surgical practice and infectious disease prevention procedures. The present findings may be useful to clinicians and the ophthalmic society members and directors and for planning the strategy for future waves of the pandemic.

Author Contributions: Conceptualization, J.K. and D.D.; methodology, J.K.; software, E.S; validation, J.K, E.S. and K.G.; formal analysis, J.K.; investigation, K.G.; resources, E.S.; data curation, N.D.; writing - original draft preparation, D.D.; writing - review and editing, J.K, D.D.; visualization, J.K.; supervision, I.O.; project administration, I.O.; All authors have read and agreed to the published version of the manuscript

Funding: This research received no external funding

Institutional Review Board Statement: Bioethics Committee of the Medical University of Białystok (APK.002.87.2021)

Data Availability Statement: In All materials and information are available from the corresponding author upon request by e-mail.

Acknowledgments: We would like to thank Ms. Marlena Puzuk for her help in searching the database.

Conflicts of Interest: The authors declare no conflict of interest.

7. Nguyen, A.X.; Gervasio, K.A.; Wu, A.Y. Differences in SARS-CoV-2 recommendations from major ophthalmology societies worldwide. BMJ Open Ophthalmol 2020, 5, e000525. https://doi.org/10.1136/bmjophth-2020-000525 
8. American Academy of Ophthalmology. Recommendations for urgent and nonurgent patient care. Available online: https://www.aao.org/headline/new-recommendations-urgent-nonurgent-patient-care (accessed on 12 July 2021).

9. Returning to ophthalmology practice. American Academy of Ophthalmology blog post. Available online: https://www.aao.org/about/governance/academy-blog/post/returning-to-ophthalmology-practice (accessed on 12 November ).

10. The Royal College of Ophthalmologists. Glaucoma Management Plans during COVID-19.; 2020.

11. Alahmadi, A.S.; Alhatlan, H.M.; Bin Helayel, H.; Khandekar, R.; Al Habash, A.; Al-Shahwan, S. Residents' perceived impact of COVID-19 on Saudi ophthalmology training programs-a survey. Clin Ophthalmol 2020, 14, 3755-3761. https://doi.org/10.2147/OPTH.S283073

12. Konopińska, J.; Obuchowska, I.; Lisowski, Ł.; Dub, N.; Dmuchowska, D.A.; Rękas, M. Impact of the COVID-19 pandemic on ophthalmic specialist training in Poland. PLoS One 2021, 16, e0257876. https://doi.org/10.1371/journal.pone.0257876

13. Caserotti, M.; Girardi, P.; Rubaltelli, E.; Tasso, A.; Lotto, L.; Gavaruzzi, T. Associations of COVID-19 risk perception with vaccine hesitancy over time for Italian residents. Soc Sci Med 2021, 272, 113688. https://doi.org/10.1016/j.socscimed.2021.113688

14. El-Saied, H.M.A.; Salah Eddin Abdelhakim, M.A. Impact of COVID-19 pandemic on young ophthalmologists in Cairo University Hospitals. Semin Ophthalmol 2020, 35, 296-306. https://doi.org/10.1080/08820538.2020.1826046

15. Silva, N.; Laiginhas, R.; Meireles. A.; Barbosa Breda, J. Impact of the COVID-19 pandemic on ophthalmology residency training in Portugal. Acta Med Port 2020, 33, 640-648. https://doi.org/10.20344/amp.14341

16. dell'Omo, R.; Filippelli, M.; Virgili, G.; Bandello, F.; Querques, G.; Lanzetta, P.; Avitabile, T.; Viola, F.; Reibaldi, M.; Semeraro, F.; et al. Effect of COVID-19-related lockdown on ophthalmic practice in Italy: A report from 39 institutional centers. Eur J Ophthalmol 2021, 11206721211002442. doi: 10.1177/11206721211002442.

17. Subathra, G.N.; Rajendrababu, S.R.; Senthilkumar, V.A.; Mani, I.; Udayakumar, B. Impact of COVID-19 on follow-up and medication adherence in patients with glaucoma in a tertiary eye care centre in south India. Indian J Ophthalmol 2021, 69, 1264-1270. https://doi.org/10.4103/ijo.IJO_164_21

18. Leung, E.H.; Flynn, H.W. Jr.; Gayer, S.; Yeh, S.; Berrocal, A.M.; Olmos de Koo, L.C.; Chen, R.W.S.; Albini, T.A.; Foroozan, R. Clinical and perioperative management in ophthalmology during the COVID-19 pandemic. Int Ophthalmol Clin 2020, 60, 141158. https://doi.org/10.1097/IIO.0000000000000310

19. Wickham, L.; Hay, G.; Hamilton, R.; Wooding, J.; Tossounis, H.; da Cruz, L.; Siriwardena, D.; Strouthidis, N. The impact of COVID policies on acute ophthalmology services-experiences from Moorfields Eye Hospital NHS Foundation Trust. Eye (Lond) 2020, 34, 1189-1192. https://doi.org/10.1038/s41433-020-0957-2

20. Tejwani, S.; Angmo, D.; Nayak, B.K.; Tejwani, S.; Angmo, D.; Nayak, B.K.; Sharma, N.; Sachdev, M.S.; Dada, T.; Sinha, R.; et al. Preferred practice guidelines for glaucoma management during COVID-19 pandemic. Indian J Ophthalmol 2020, 68, $1277-1280$. https://doi.org/10.4103/ijo.IJO_1724_20

21. Parikh, R.S.; George, R.; Shantha, B.; Parikh, S.R.; Vijaya, L. Comments on: Preferred practice guidelines for glaucoma management during COVID-19 pandemic. Indian J Ophthalmol 2020, 68, 2311-2312. https://doi.org/10.4103/ijo.IJO_2255_20

22. Vinod, K.; Sidoti, P.A. Glaucoma care during the coronavirus disease 2019 pandemic. Curr Opin Ophthalmol $2021,32,75-82$. https://doi.org/10.1097/ICU.0000000000000730

23. Shabto, J.M.; De Moraes, C.G.; Cioffi, G.A.; Liebmann, J.M. Review of hygiene and disinfection recommendations for outpatient glaucoma care: a COVID era update. J Glaucoma 2020, 29, 409-416. https://doi.org/10.1097/IJG.0000000000001540

24. Rajendrababu, S.; Duraoi, I.; Mani, I.; Ramasamy, K.S.; Shukla, A.G.; Robin, A.L. Urgent and emergent glaucoma care during the COVID-19 pandemic: an analysis at a tertiary care hospital in South India. Indian J Ophthalmol 2021, 69, $2215-2221$. https://doi.org/10.4103/ijo.IJO_635_21

25. Holland, L.J.; Kirwan, J.F.; Mercieca, K.J. Effect of COVID-19 pandemic on glaucoma surgical practices in the UK. Br J Ophthalmol 2021. 0, 1-5 https://doi.org/10.1136/bjophthalmol-2021-319062

26. Du, H.; Zhang, M.; Zhang, H.; Sun, X. Practical experience on emergency ophthalmic surgery during the prevalence of COVID19. Graefes Arch Clin Exp Ophthalmol 2020, 258, 1831-1833. https://doi.org/10.1016/j.socscimed.2021.113688

27. Formichella, P.; Annoh, R.; Zeri, F.; Tatham, A.J. The role of the disc damage likelihood scale in glaucoma detection by community optometrists. Ophthalmic Physiol Opt 2020, 40, 752-759. https://doi.org/10.1111/opo.12734

28. Wong, R.; Bannerjee, P.; Kumaran, N. Aerosol generating procedures in intraocular surgery. Eye (Lond) 2021, $35,1504-1505$. https://doi.org/10.1038/s41433-020-0997-7

29. Gedde, S.J.; Feuer, W.J.; Shi, W.; Lim, K.S.; Barton, K.; Goyal, S.; Ahmed, I.I.K.; Brandt, J.; Primary Tube Versus Trabeculectomy Study Group. Treatment outcomes in the primary tube versus trabeculectomy study after 1 year of follow-up. Ophthalmology 2018, 125, 650-663. https://doi.org/10.1016/j.ophtha.2018.02.003

30. Mylona, I.; Dermenoudi, M.; Glynatsis, N.M.; Glynatsis, M.N. Patient adherence to glaucoma treatment during the COVID-19 pandemic. Cureus 2021, 13, e15545. https://doi.org/10.7759/cureus.15545

31. European Glaucoma Society Terminology and Guidelines for Glaucoma, 5th Edition. Br J Ophthalmol 2021, 105(Suppl 1), 1-169. https://doi.org/10.1136/bjophthalmol-2021-egsguidelines 
A. 